Kragujevac Journal of Mathematics

Volume 44(4) (2020), PAges 593-601.

\title{
JOHNSON PSEUDO-CONTRACTIBILITY AND PSEUDO-AMENABILITY OF $\theta$-LAU PRODUCT
}

\author{
M. ASKARI-SAYAH ${ }^{1}$, A. POURABBAS ${ }^{1}$, AND A. SAHAMI $^{2}$
}

\begin{abstract}
Given Banach algebras $A$ and $B$ and $\theta \in \Delta(B)$. We shall study the Johnson pseudo-contractibility and pseudo-amenability of the $\theta$-Lau product $A \times{ }_{\theta} B$. We show that if $A \times{ }_{\theta} B$ is Johnson pseudo-contractible, then both $A$ and $B$ are Johnson pseudo-contractible and $A$ has a bounded approximate identity. In some particular cases, a complete characterization of Johnson pseudo-contractibility of $A \times_{\theta} B$ is given. Also, we show that pseudo-amenability of $A \times_{\theta} B$ implies the approximate amenability of $A$ and pseudo-amenability of $B$.
\end{abstract}

\section{INTRODUCTION}

Let $A$ and $B$ be two Banach algebras and $\theta \in \Delta(B)$, where $\Delta(B)$ is the character space of $B$. Then the Banach space $A \times B$ with the product

$$
(a, b)(c, d)=(a c+\theta(d) a+\theta(b) c, b d), \quad a, c \in A, b, d \in B,
$$

and $\ell^{1}$-norm becomes a Banach algebra, which is called the $\theta$-Lau product of $A$ and $B$ which is denoted by $A \times_{\theta} B$. The $\theta$-Lau product was first introduce by A. T. Lau [14] for $F$-algebras. Recently, this product was extended to general Banach algebras by M. Monfared [15] for every Banach algebras $A$ and $B$ and every character $\theta \in \Delta(B)$. One may regard $A(B)$ as a closed two sided ideal (Banach subalgebra) of $A \times{ }_{\theta} B$ by identifying it with $A \times\{0\}(\{0\} \times B)$, respectively. Therefore, if there is no ambiguity, we may simply write $a(b)$ instead of $(a, 0)((0, b))$ for every $a \in A(b \in B)$, respectively. Monfared studied several properties of $A \times_{\theta} B$ including semisimpility, Arens regularity, existence of approximate identity and amenability. We recall that the concept of an amenable Banach algebra was introduced by Johnson in 1972. Indeed,

Key words and phrases. $\theta$-Lau product, Johnson pseudo-contractibility, pseudo-amenability. 2010 Mathematics Subject Classification. Primary: 46H05, 46H20. Secondary: 43A20.

DOI 10.46793/KgJMat2004.593A

Received: May 20, 2018.

Accepted: July 21, 2018. 
a Banach algebra $A$ is called amenable if there is an element $M \in\left(A \otimes_{p} A\right)^{* *}$ such that $a \cdot M=M \cdot a$ and $\pi_{A}^{* *}(M) a=a$ for every $a \in A$, where $\pi: A \otimes_{p} A \rightarrow A$ is the product morphism and $A \otimes_{p} A$ is the projective tensor product of $A$. Motivated by this construction of Johnson, some authors introduce several modifications of this notion by relaxing some conditions in different versions of definitions of amenability. The notion of pseudo-amenability was introduced by F. Ghahramani and Y. Zhang [13]. A Banach algebra $A$ is called pseudo-amenable if there is a net $\left(m_{\alpha}\right) \subseteq A \otimes_{p} A$ such that $a \cdot m_{\alpha}-m_{\alpha} \cdot a \rightarrow 0$ and $\pi_{A}\left(m_{\alpha}\right) a \rightarrow a$ for every $a \in A$. The concept of approximately amenable Banach algebras was introduced by F. Ghahramani and R. J. Loy in [11], see also [12]. A Banach algebra $A$ is called approximately amenable if there are nets $\left(M_{\alpha}\right) \subseteq A \otimes_{p} A,\left(F_{\alpha}\right) \subseteq A$ and $\left(G_{\alpha}\right) \subseteq A$ such that for every $a \in A$

(i) $a \cdot M_{\alpha}-M_{\alpha} \cdot a+F_{\alpha} \otimes a-a \otimes G_{\alpha} \rightarrow 0$;

(ii) $a F_{\alpha} \rightarrow a, G_{\alpha} a \rightarrow a$ and

(iii) $\pi_{A}\left(M_{\alpha}\right) a-F_{\alpha} a-G_{\alpha} a \rightarrow 0$.

Recently the second and third authors [19] have defined a new concept related to amenability called Johnson pseudo-contractibility. Indeed, a Banach algebra $A$ is called Johnson pseudo-contractible if there is a not necessarily bounded net $\left(M_{\alpha}\right) \subseteq$ $\left(A \otimes_{p} A\right)^{* *}$ such that $a \cdot M_{\alpha}=M_{\alpha} \cdot a$ and $\pi_{A}^{* *}\left(M_{\alpha}\right) a-a \rightarrow 0$ for every $a \in A$.

In the Section 2 we deal with Johnson pseudo-contractible Banach algebras. We show that if $A \times_{\theta} B$ is Johnson pseudo-contractible, then $A$ is Johnson pseudocontractible and has a bounded approximate identity and $B$ is Johnson pseudocontractible. Moreover, we show that in particular cases, for example when $A$ is Arens regular and weakly sequentially complete or when $A$ is a dual Banach algebra, Johnson pseudo-contractibility of $A \times_{\theta} B$ is equivalent with amenability of $A$ and Johnson pseudo-contractibility of $B$. Some example are given at the end of the section.

In the Section 3 we focus on pseudo-amenability of $A \times_{\theta} B$. Pseudo-amenability of $A \times{ }_{\theta} B$ was studied by E. Ghaderi et al. [10]. They showed that pseudo-amenability of $A \times_{\theta} B$ implies pseudo-amenability of $B$, and implies pseudo-amenability of $A$ whenever $A$ has a bounded approximate identity. We show that the existence of bounded approximate identity in this result is not a necessary condition. Indeed, we show that if $A \times{ }_{\theta} B$ is pseudo-amenable, then $A$ is approximately amenable and $B$ is pseudo-amenable.

\section{Johnson Pseudo-Contractibility of $A \times_{\theta} B$}

We state a result from [2] that will be used frequently in this section.

Theorem 2.1. Let $A$ be a Johnson pseudo-contractible Banach algebra with an identity. Then $A$ is amenable.

Lemma 2.1. Let $A$ be a Johnson pseudo-contractible Banach algebra and let I be a two sided closed ideal of $A$. If I has a bounded approximate identity, then I is Johnson pseudo-contractible. 
Proof. By hypothesis there is a net $\left(M_{\alpha}\right) \subseteq\left(A \otimes_{p} A\right)^{* *}$ such that $a \cdot M_{\alpha}=M_{\alpha} \cdot a$ and $\pi_{A}^{* *}\left(M_{\alpha}\right) a-a \rightarrow 0$ for every $a \in A$. Let $\left(e_{\beta}\right)$ be a bounded approximate identity for $I$ and let $E$ be a weak* cluster point of $\left(e_{\beta}\right)$ in $I^{* *}$. Then by setting $\left(N_{\alpha}\right)=$ $\left(E \cdot M_{\alpha} \cdot E\right) \subseteq\left(I \otimes_{p} I\right)^{* *}$, we have

$$
x \cdot N_{\alpha}=N_{\alpha} \cdot x
$$

and

$$
\pi_{I}^{* *}\left(N_{\alpha}\right) x=\pi_{A}^{* *}\left(E \cdot M_{\alpha} \cdot E\right) x=\pi_{A}^{* *}\left(M_{\alpha}\right) x \rightarrow x
$$

for every $x \in I$. It follows that $I$ is Johnson pseudo-contractible.

Theorem 2.2. Let $A$ and $B$ be two Banach algebras and $\theta \in \Delta(B)$. If $A \times_{\theta} B$ is Johnson pseudo-contractible, then the following statements hold.

(a) A is Johnson pseudo-contractible and has a bounded approximate identity.

(b) B is Johnson pseudo-contractible.

Proof. Suppose that $\Phi:\left(A \times_{\theta} B\right) \otimes_{p}\left(A \times_{\theta} B\right) \rightarrow A \times_{\theta} B$ is the linear map determined by

$$
\Phi((a, b) \otimes(c, d))=\theta(d)(a, b), \quad a, c \in A, b, d \in B .
$$

Let $\left(U_{\alpha}\right) \subseteq\left(\left(A \times_{\theta} B\right) \otimes_{p}\left(A \times_{\theta} B\right)\right)^{* *}$ be such that

$$
(a, b) \cdot U_{\alpha}=U_{\alpha} \cdot(a, b), \quad \pi_{A \times{ }_{\theta} B}^{* *}\left(U_{\alpha}\right)(a, b) \rightarrow(a, b),
$$

for every $a \in A$ and $b \in B$. Then by Goldstine's theorem for every $\alpha$ there exists a net $\left(u_{\alpha_{\beta}}\right)$ in $\left(A \times_{\theta} B\right) \otimes_{p}\left(A \times_{\theta} B\right)$ such that $w^{*}-\lim _{\beta} u_{\alpha_{\beta}}=U_{\alpha}$. Suppose that $u_{\alpha_{\beta}}=\sum_{i=1}^{\infty}\left(a_{i}^{\alpha_{\beta}}, b_{i}^{\alpha_{\beta}}\right) \otimes\left(c_{i}^{\alpha_{\beta}}, d_{i}^{\alpha_{\beta}}\right)$ for sequences $\left(a_{i}^{\alpha_{\beta}}\right),\left(c_{i}^{\alpha_{\beta}}\right) \subseteq A$ and $\left(b_{i}^{\alpha_{\beta}}\right),\left(d_{i}^{\alpha_{\beta}}\right) \subseteq B$, where $\sum_{i=1}^{\infty}\left\|\left(a_{i}^{\alpha_{\beta}}, b_{i}^{\alpha_{\beta}}\right)\right\| \cdot\left\|\left(c_{i}^{\alpha_{\beta}}, d_{i}^{\alpha_{\beta}}\right)\right\|<\infty$. Note that $\theta$ has an extension $\tilde{\theta} \in \Delta\left(B^{* *}\right)$ given by $\tilde{\theta}(F)=F(\theta)$ for every $F \in B^{* *}$. Since $\Phi$ and $\theta$ are bounded, $\Phi^{* *}$ and $\tilde{\theta}$ are weak* continuous maps. Now we have

$$
\begin{aligned}
\left\langle(0, \tilde{\theta}), \Phi^{* *}\left(U_{\alpha}\right)\right\rangle & =w^{*}-\lim _{\beta}\left\langle(0, \theta), \Phi\left(u_{\alpha_{\beta}}\right)\right\rangle \\
& =w^{*}-\lim _{\beta} \sum_{i=1}^{\infty} \theta\left(b_{i}^{\alpha_{\beta}}\right) \theta\left(b_{i}^{\alpha_{\beta}}\right) \\
& =w^{*}-\lim _{\beta}\left\langle(0, \theta), \pi_{A \times_{\theta} B}\left(u_{\alpha_{\beta}}\right)\right\rangle \\
& =\left\langle(0, \tilde{\theta}), \pi_{A \times_{\theta} B}^{* *}\left(U_{\alpha}\right)\right\rangle \rightarrow 1 .
\end{aligned}
$$

Set $\Phi^{* *}\left(U_{\alpha}\right)=\left(\phi_{\alpha}, \psi_{\alpha}\right)$, where $\phi_{\alpha} \in A^{* *}$ and $\psi_{\alpha} \in B^{* *}$. We can see that $\tilde{\theta}\left(\psi_{\alpha}\right) \rightarrow 1$. Take $\alpha_{0}$ such that $\tilde{\theta}\left(\psi_{\alpha_{0}}\right) \neq 0$, for every $a \in A$ we have

$$
a \Phi^{* *}\left(U_{\alpha_{0}}\right)=\Phi^{* *}\left(a \cdot U_{\alpha_{0}}\right)=\Phi^{* *}\left(U_{\alpha_{0}} \cdot a\right)=0 .
$$

Also, we have

$$
a \Phi^{* *}\left(U_{\alpha_{0}}\right)=(a, 0)\left(\phi_{\alpha_{0}}, \psi_{\alpha_{0}}\right)=\left(a \phi_{\alpha_{0}}+\tilde{\theta}\left(\psi_{\alpha_{0}}\right) a, 0\right)
$$


Therefore $a \phi_{\alpha_{0}}+\tilde{\theta}\left(\psi_{\alpha_{0}}\right) a=0$, so $a\left(-\tilde{\theta}\left(\psi_{\alpha_{0}}\right)^{-1} \phi_{\alpha_{0}}\right)=a$, where $-\tilde{\theta}\left(\psi_{\alpha_{0}}\right)^{-1} \phi_{\alpha_{0}} \in A^{* *}$. This shows that $A$ has a bounded right approximate identity. A similar argument shows that $A$ has a bounded left approximate identity. It follows that $A$ has a bounded approximate identity. Since $A$ is a two sided closed ideal of $\left(A \times_{\theta} B\right)$ and has a bounded approximate identity, by Lemma 2.1 it is Johnson pseudo-contractible.

It is well known that $\left(A \times_{\theta} B\right) / A \cong B$ and there is a surjective homomorphism from $A \times{ }_{\theta} B$ onto $\left(A \times_{\theta} B\right) / A$. So, [19, Proposition 2.9] implies Johnson pseudocontractibility of $B$.

We remark that the converse of the previous theorem does not hold in general. For example, $A(H)$, the Fourier algebra on the integer Heisenberg group $H$, is Johnson pseudo-contractible and has a bounded approximate identity and $M(H)$, the measure algebra over $H$, is Johnson pseudo-contractible ( $H$ is discrete and amenable). But $A(H) \times_{\theta} M(H)$ is not Johnson pseudo-contractible for every $\theta \in \Delta(M(H))$. Indeed, $A(H) \times_{\theta} M(H)$ has an identity [15, Proposition 2.3]. If $A(H) \times_{\theta} M(H)$ is Johnson pseudo-contractible, then, by Theorem 2.1, $A(H) \times_{\theta} M(H)$ is amenable and [15, page 285] implies the amenability of $A(H)$. It gives a contradiction that $H$ has an abelian subgroup of finite index, see [9, Theorem 2.3].

From [15, page 285] and Theorem 2.1, we have the following corollary.

Corollary 2.1. If $B$ has an identity, then the following statements are equivalent:

(a) $A \times_{\theta} B$ is Johnson pseudo-contractible;

(b) $A \times_{\theta} B$ is amenable;

(c) $A$ and $B$ are amenable.

Corollary 2.2. If $A$ has an identity, then $A \times{ }_{\theta} B$ is Johnson pseudo-contractible if and only if $A$ is amenable and $B$ is Johnson pseudo-contractible.

Proof. In view of [3] $A \times_{\theta} B$ is nothing but the $\ell^{1}$-direct sum $A \oplus B$ with coordinatewise product whenever $A$ has an identity. If $A$ is amenable and $B$ is Johnson pseudocontractible, then $A \oplus B$ is Johnson pseudo-contractible by [19, Theorem 2.11]. The converse comes immediately from Theorem 2.2 and Theorem 2.1.

A Banach algebra $A$ is called dual if it is a dual space such that multiplication in $A$ is separately $w^{*}$-continuous. It is well known that a dual Banach algebra with a bounded approximate identity has an identity [18, Proposition 1.2], so we have the following corollary from Theorem 2.2 and Corollary 2.2.

Corollary 2.3. Let $B$ be a Banach algebra and let $A$ be a dual Banach algebra and $\theta \in \Delta(B)$. Then $A \times_{\theta} B$ is Johnson pseudo-contractible if and only if $A$ is amenable and $B$ is Johnson pseudo-contractible.

A Banach algebra $A$ is called Arens regular if the first and the second Arens products on $A^{* *}$ coincide. Also, a Banach algebra $A$ is called weakly sequentially complete if every weakly Cauchy sequence in $A$ is weakly convergent. 
Proposition 2.1. Suppose that $A$ and $B$ are two Banach algebras and $\theta \in \Delta(B)$. If $A$ is Arens regular and weakly sequentially complete, then $A \times_{\theta} B$ is Johnson pseudocontractible if and only if

(a) $A$ is amenable and has an identity;

(b) B is Johnson pseudo-contractible.

Proof. If $A \times_{\theta} B$ is Johnson pseudo-contractible, then, by Theorem 2.1, $A$ has a bounded approximate identity. Using Ülger theorem [4, Theorem 2.9.39], $A$ has an identity. Now apply Corollary 2.2.

It seems that Johnson pseudo-contractibility of $A \times_{\theta} B$ is related with amenability of $A$. We believe that Corollary 2.2 holds without the assumption that $A$ has an identity. However, it remains as a conjecture. We left it as an open problem in the following questions.

Question 1. Does Johnson pseudo-contractibility of $A \times_{\theta} B$ implies the amenability of $A$ ?

Question 2. Suppose that $A$ is an amenable Banach algebra and $B$ is a Johnson pseudocontractible Banach algebra and $\theta \in \Delta(B)$. Is $A \times_{\theta} B$ a Johnson pseudo-contractible Banach algebra?

We finish this section with some examples. First we recall some concepts and notations from semigroup theory. A semigroup $S$ is called regular if for every $s \in S$ there exists an element $t \in S$ such that $s t s=s$ and $t s t=t$. A semigroup $S$ is an inverse semigroup if for every $s \in S$ there exists a unique element $t \in S$ such that $s t s=s$ and $t s t=t$. The set of idempotents of a semigroup $S$ is denoted by $E(S)$, which is a partially ordered set with the following order

$$
p \leq q \Leftrightarrow p=p q=q p, \quad p, q \in E(S) .
$$

For $p \in E(S)$, we set $(p]=\{x: x \leq p\}$. An inverse semigroup $S$ is called uniformly locally finite if $\sup \{|(p]|: p \in E(S)\}<\infty$. It is well known that the discrete semigroup algebra $\ell^{1}(S)$ is weakly sequentially complete [4, Theorem A.4.4]. Our main reference for semigroup theory is [5].

Example 2.1. Suppose that $B$ is a Banach algebra and $\theta \in \Delta(B)$.

(i) Let $S$ be a uniformly locally finite inverse semigroup. Then Johnson pseudocontractibility of $\ell^{1}(S) \times_{\theta} B$ implies that $\ell^{1}(S)$ is Johnson pseudo-contractible and has a bounded approximate identity. From [16, Proposition 2.1] $E(S)$ must be finite and from [20, Theorem 2.3] every maximal subgroup of $S$ is amenable, in other word $\ell^{1}(S)$ is amenable, see [7].

(ii) Suppose that $S$ is regular and $\ell^{1}(S)$ is Arens regular. If $\ell^{1}(S) \times_{\theta} B$ is Johnson pseudo-contractible, then, by Proposition $2.1, \ell^{1}(S)$ is amenable and has an identity. So, by [7], $E(S)$ is finite. Now [5, Theorem 12.2] implies that $S$ is a unital finite semigroup. Indeed, $\ell^{1}(S) \times_{\theta} B$ is Johnson pseudo-contractible if and only if $S$ is a unital finite semigroup and $B$ is Johnson pseudo-contractible. 
Example 2.2. Using [8, Theorem 3.1] one can see that $M_{I}(\mathbb{C})$ (the Banach algebra of $I \times I$-matrices over $\mathbb{C}$, with finite $\ell^{1}$-norm and matrix multiplication) has no bounded approximate identity unless $I$ is finite, but in this case $M_{I}(\mathbb{C})$ is amenable and has an identity. So, for Banach algebra $B$ and $\theta \in \Delta(B), M_{I}(\mathbb{C}) \times_{\theta} B$ is Johnson pseudocontractible if and only if $I$ is finite and $B$ is Johnson pseudo-contractible.

A linear subspace $S^{1}(G)$ of $L^{1}(G)$ is said to be a Segal algebra on $G$ if it satisfies the following conditions:

(i) $S^{1}(G)$ is dense in $L^{1}(G)$;

(ii) $S^{1}(G)$ with a norm $\|\cdot\|_{S^{1}(G)}$ is a Banach space and $\|f\|_{L^{1}(G)} \leq\|f\|_{S^{1}(G)}$ for every $f \in S^{1}(G)$;

(iii) $S^{1}(G)$ is left translation invariant (that is, $L_{y} f \in S^{1}(G)$ for every $f \in S^{1}(G)$ and $y \in G)$ and the map $y \mapsto L_{y}(f)$ from $G$ into $S^{1}(G)$ is continuous, where $L_{y}(f)(x)=f\left(y^{-1} x\right)$

(iv) $\left\|L_{y}(f)\right\|_{S^{1}(G)}=\|f\|_{S^{1}(G)}$, for every $f \in S^{1}(G)$ and $y \in G$.

Example 2.3. Suppose that $B$ is a Banach algebra and $\theta \in \Delta(B)$. Let $S^{1}(G)$ be a Segal algebra on $G$. If $S^{1}(G) \times_{\theta} B$ is Johnson pseudo-contractible, then $S^{1}(G)=L^{1}(G)$.

\section{Pseudo-Amenability of $A \times_{\theta} B$}

Remark 3.1. Note that if $U \in\left(A \times_{\theta} B\right) \otimes_{p}\left(A \times_{\theta} B\right)$, then there are $M \in A \otimes_{p} A$, $N \in A \otimes_{p} B, L \in B \otimes_{p} A$ and $H \in B \otimes_{p} B$ such that

$$
U=M+N+L+H
$$

and

$$
\|U\|_{\left(A \times_{\theta} B\right) \otimes_{p}\left(A \times_{\theta} B\right)}=\|M\|_{A \otimes_{p} A}+\|N\|_{A \otimes_{p} B}+\|L\|_{B \otimes_{p} A}+\|H\|_{B \otimes_{p} B} .
$$

Theorem 3.1. Suppose that $A$ and $B$ are Banach algebras and $\theta \in \Delta(B)$. If $A \times{ }_{\theta} B$ is pseudo-amenable, then

(a) $A$ is approximate amenable and

(b) $B$ is pseudo-amenable.

Proof. It is well known that $\left(A \times_{\theta} B\right) / A \cong B$ and there is a surjective homomorphism from $A \times_{\theta} B$ onto $\left(A \times_{\theta} B\right) / A$. So [13, Proposition 2.2] implies pseudo-amenability of $B$.

By assumption there is a net $\left(U_{\alpha}\right) \subseteq\left(A \times_{\theta} B\right) \otimes_{p}\left(A \times_{\theta} B\right)$ such that

$$
(x, y) \cdot U_{\alpha}-U_{\alpha} \cdot(x, y) \rightarrow 0, \quad \pi\left(U_{\alpha}\right)(x, y) \rightarrow(x, y),
$$

for every $x \in A, y \in B$. Particularly for every $x \in A$ we have

$$
x \cdot U_{\alpha}-U_{\alpha} \cdot x \rightarrow 0, \quad \pi\left(U_{\alpha}\right) x \rightarrow x .
$$

Suppose that $U_{\alpha}=\sum_{i=1}^{\infty}\left(a_{i}^{\alpha}, b_{i}^{\alpha}\right) \otimes\left(c_{i}^{\alpha}, d_{i}^{\alpha}\right)$ for sequences $\left(a_{i}^{\alpha}\right),\left(c_{i}^{\alpha}\right) \subseteq A$ and $\left(b_{i}^{\alpha}\right),\left(d_{i}^{\alpha}\right) \subseteq$ $B$, where $\sum_{i=1}^{\infty}\left\|\left(a_{i}^{\alpha}, b_{i}^{\alpha}\right)\right\| \cdot\left\|\left(c_{i}^{\alpha}, d_{i}^{\alpha}\right)\right\|<\infty$. Set $M_{\alpha}=\sum_{i=1}^{\infty} a_{i}^{\alpha} \otimes c_{i}^{\alpha}, F_{\alpha}=-\sum_{i=1}^{\infty} \theta\left(d_{i}^{\alpha}\right) a_{i}^{\alpha}$, 
$G_{\alpha}=-\sum_{i=1}^{\infty} \theta\left(b_{i}^{\alpha}\right) c_{i}^{\alpha}$ and $H_{\alpha}=\sum_{i=1}^{\infty} b_{i}^{\alpha} \otimes d_{i}^{\alpha}$. One can easily see that

$$
\pi_{A \times{ }_{\theta} B}\left(U_{\alpha}\right)=\left(\pi_{A}\left(M_{\alpha}\right)-F_{\alpha}-G_{\alpha}, \pi_{B}\left(H_{\alpha}\right)\right) .
$$

For an arbitrary element $b$ in $B$, we have

$$
\pi_{A \times_{\theta} B}\left(U_{\alpha}\right)(0, b)=\left(\theta(b)\left(\pi_{A}\left(M_{\alpha}\right)-F_{\alpha}-G_{\alpha}\right), \pi_{B}\left(H_{\alpha}\right) b\right) \rightarrow(0, b),
$$

so

$$
\pi_{A}\left(M_{\alpha}\right)-F_{\alpha}-G_{\alpha} \rightarrow 0, \quad \theta\left(\pi_{B}\left(H_{\alpha}\right)\right) \rightarrow 1
$$

Note that

$$
\begin{aligned}
x \cdot U_{\alpha}= & \sum_{i=1}^{\infty}(x, 0)\left(a_{i}^{\alpha}, 0\right) \otimes\left(c_{i}^{\alpha}, 0\right)+\sum_{i=1}^{\infty}(x, 0)\left(0, b_{i}^{\alpha}\right) \otimes\left(c_{i}^{\alpha}, 0\right) \\
& +\sum_{i=1}^{\infty}(x, 0)\left(a_{i}^{\alpha}, 0\right) \otimes\left(0, d_{i}^{\alpha}\right)+\sum_{i=1}^{\infty}(x, 0)\left(0, b_{i}^{\alpha}\right) \otimes\left(0, d_{i}^{\alpha}\right) \\
= & x \cdot\left(\sum_{i=1}^{\infty}\left(a_{i}^{\alpha} \otimes c_{i}^{\alpha}\right)\right)+\sum_{i=1}^{\infty}\left(x \otimes \theta\left(b_{i}^{\alpha}\right) c_{i}^{\alpha}\right)+\sum_{i=1}^{\infty}\left(x a_{i}^{\alpha} \otimes d_{i}^{\alpha}\right)+\sum_{i=1}^{\infty}\left(\theta\left(b_{i}^{\alpha}\right) x \otimes d_{i}^{\alpha}\right) \\
= & x \cdot M_{\alpha}-x \otimes G_{\alpha}+\sum_{i=1}^{\infty}\left(x a_{i}^{\alpha} \otimes d_{i}^{\alpha}\right)+\sum_{i=1}^{\infty}\left(\theta\left(b_{i}^{\alpha}\right) x \otimes d_{i}^{\alpha}\right) .
\end{aligned}
$$

Similarly we have

$$
U_{\alpha} \cdot x=M_{\alpha} \cdot x-F_{\alpha} \otimes x+\sum_{i=1}^{\infty}\left(b_{i}^{\alpha} \otimes c_{i}^{\alpha} x\right)+\sum_{i=1}^{\infty}\left(b_{i}^{\alpha} \otimes \theta\left(d_{i}^{\alpha}\right) x\right) .
$$

From (3.2), (3.3) and (3.1), by using Remark 3.1 we obtain

(a) $x \cdot M_{\alpha}-M_{\alpha} \cdot x+F_{\alpha} \otimes x-x \otimes G_{\alpha} \rightarrow 0$;

(b) $\sum_{i=1}^{\infty}\left(x a_{i}^{\alpha} \otimes d_{i}^{\alpha}\right)+\sum_{i=1}^{\infty}\left(\theta\left(b_{i}^{\alpha}\right) x \otimes d_{i}^{\alpha}\right) \rightarrow 0$;

(c) $\sum_{i=1}^{\infty}\left(b_{i}^{\alpha} \otimes c_{i}^{\alpha} x\right)+\sum_{i=1}^{\infty}\left(b_{i}^{\alpha} \otimes \theta\left(d_{i}^{\alpha}\right) x\right) \rightarrow 0$.

Define a bounded linear map $\phi: A \otimes_{p} B \rightarrow A$ by $\phi(a \otimes b)=\theta(b) a$. From (b) we have

$$
\begin{aligned}
-x F_{\alpha}+\theta\left(\pi_{B}\left(H_{\alpha}\right)\right) x & =x \sum_{i=1}^{\infty} \theta\left(d_{i}^{\alpha}\right) a_{i}^{\alpha}+\sum_{i=1}^{\infty} \theta\left(b_{i}^{\alpha} d_{i}^{\alpha}\right) x \\
& =\phi\left(\sum_{i=1}^{\infty}\left(x a_{i}^{\alpha} \otimes d_{i}^{\alpha}\right)+\sum_{i=1}^{\infty}\left(\theta\left(b_{i}^{\alpha}\right) x \otimes d_{i}^{\alpha}\right)\right) \rightarrow 0
\end{aligned}
$$

now $\theta\left(\pi_{B}\left(H_{\alpha}\right)\right) \rightarrow 1$ implies that $x F_{\alpha} \rightarrow x$. Similarly, by using (c) we have $G_{\alpha} x \rightarrow x$. So we find $\left(M_{\alpha}\right) \subseteq A \otimes_{p} A,\left(F_{\alpha}\right) \subseteq A$ and $\left(G_{\alpha}\right) \subseteq A$ such that
(a) $x \cdot M_{\alpha}-M_{\alpha} \cdot x+F_{\alpha} \otimes x-x \otimes G_{\alpha} \rightarrow 0$;
(b) $x F_{\alpha} \rightarrow x, \quad G_{\alpha} x \rightarrow x$;
(c) $\pi_{A}\left(M_{\alpha}\right) x-F_{\alpha} x-G_{\alpha} x \rightarrow 0$, 
for every $x \in A$. It follows that $A$ is approximately amenable.

Example 3.1. Let $S$ be a uniformly locally finite inverse semigroup and let $B$ be a Banach algebra and $\theta \in \Delta(B)$. If $\ell^{1}(S) \times_{\theta} B$ is pseudo-amenable, then by Theorem 3.1 $\ell^{1}(S)$ is approximately amenable. Theorem 4.3 of [17] shows that $\ell^{1}(S)$ is amenable.

Example 3.2. Let $G=S U(2)$ be the $2 \times 2$ unitary group, and suppose that $S^{1}(G) \neq$ $L^{1}(G)$ is a Segal algebra on $G$. In [1] Alaghmandan showed that $S^{1}(G)$ is not approximately amenable. Thus, by Theorem $3.1, S^{1}(G) \times_{\theta} B$ is not pseudo-amenable for every Banach algebra $B$ and $\theta \in \Delta(B)$.

Example 3.3. Let $G$ be an infinite abelian compact group and let $B$ be a Banach algebra and $\theta \in \Delta(B)$. We claim that $L^{2}(G) \times_{\theta} B$ is not pseudo-amenable. To see this, suppose that $L^{2}(G) \times_{\theta} B$ is pseudo-amenable. Then Theorem 3.1 implies that $L^{2}(G)$ is approximately amenable. But by the Plancherel theorem $L^{2}(G)$ is isometrically isomorphism to $\ell^{2}(\hat{G})$, where $\hat{G}$ is the dual group of $G$ and $\ell^{2}(\hat{G})$ is equipped with the pointwise product. So $\ell^{2}(\hat{G})$ is approximately amenable which is a contradiction with the main result of $[6]$.

Acknowledgements. The authors are grateful to the referee for carefully reading the paper, pointing out a number of misprints and for some helpful comments.

\section{REFERENCES}

[1] M. Alaghmandan, Approximate amenability of Segal algebras, J. Aust. Math. Soc. 95(1) (2013), 20-35.

[2] M. Askari-Sayah, A. Pourabbas and A. Sahami, Johnson pseudo-contractibility of certain Banach algebras and their nilpotent ideals, Analysis Mathematica (to appear).

[3] Y. Choi, Triviality of the generalised Lau product associated to a Banach algebra homomorphism, Bull. Aust. Math. Soc. 94(2) (2016), 286-289.

[4] H. G. Dales, Banach Algebras and Automatic Continuity, London Mathematical Society Monographs, New Series 24, The Clarendon Press, Oxford University Press, New York, 2000.

[5] H. G. Dales, A. T. M. Lau and D. Strauss, Banach Algebras on Semigroups and on Their Compactifications, Memoirs of the American Mathematical Society 205(996), American Mathematical Society, Providence, 2010.

[6] H. G. Dales, R. J. Loy, and Y. Zhang, Approximate amenability for Banach sequence algebras, Studia Math. 177(1) (2006), 81-96.

[7] J. Duncan and A. L. T. Paterson, Amenability for discrete convolution semigroup algebras, Math. Scand. 66(1) (1990), 141-146.

[8] G. H. Esslamzadeh, Double centralizer algebras of certain Banach algebras, Monatsh. Math. $\mathbf{1 4 2 ( 3 )}$ (2004), 193-203.

[9] B. Forrest and V. Runde, Amenability and weak amenability of the Fourier algebra, Math. Z. 250(4) (2005), 731-744.

[10] E. Ghaderi, R. Nasr-Isfahani and M. Nemati, Pseudo-amenability and pseudo-contractibility for certain products of Banach algebras, Math. Slovaca 66(6) (2016), 1367-1374.

[11] F. Ghahramani and R. J. Loy, Generalized notions of amenability, J. Funct. Anal. 208(1) (2004), $229-260$. 
[12] F. Ghahramani, R. J. Loy and Y. Zhang, Generalized notions of amenability II, J. Funct. Anal. 254(7) (2008), 1776-1810.

[13] F. Ghahramani and Y. Zhang, Pseudo-amenable and pseudo-contractible Banach algebras, Math. Proc. Cambridge Philos. Soc. 142(1) (2007), 111-123.

[14] A. Lau, Analysis on a class of Banach algebras with applications to harmonic analysis on locally compact groups and semigroups, Fund. Math. 118(3) (1983), 161-175.

[15] M. Monfared, On certain products of Banach algebras with applications to harmonic analysis, Studia Math. 178(3) (2007), 277-294.

[16] P. Ramsden, Biflatness of semigroup algebras, Semigroup Forum 79(3) (2009), 515-530.

[17] M. Rostami, A. Pourabbas and M. Essmaili, Approximate amenability of certain inverse semigroup algebras, Acta Math. Sci. Ser. B Engl. Ed. 33(2) (2013), 565-577.

[18] V. Runde, Amenability for dual Banach algebras, Studia Math. 148(1) (2001), 47-66.

[19] A. Sahami and A. Pourabbas, Johnson pseudo-contractibility of various classes of Banach algebras, Bull. Belg. Math. Soc. Simon Stevin 25(2) (2018), 171-182.

[20] A. Sahami and A. Pourabbas, Johnson pseudo-contractibility of certain semigroup algebras, Semigroup Forum 97(2) (2018), 203-213.

${ }^{1}$ Faculty of Mathematics and Computer Science,

AMirkabir University of TeChNOLOGy,

424 Hafez Avenue, 15914 Tehran, Iran

Email address: mehdi17@aut.ac.ir

Email address: arpabbas@aut.ac.ir

${ }^{2}$ Department of Mathematics, Faculty of Basic Sciences,

ILAM UNIVERSITY,

P.O. Box 69315-516, Ilam, IrAN

Email address: a.sahami@ilam.ac.ir 